\title{
A NEW PERSPECTIVE FOR MULTITEMPORAL SAR DATA ANALYSIS
}

\author{
Donato Amitrano, Gerardo Di Martino, Antonio Iodice, Daniele Riccio and Giuseppe Ruello
}

\author{
Department of Electrical Engineering and Information Technology, \\ University of Napoli Federico II, via Claudio 21, 80125 Napoli, Italy
}

\section{ABSTRACT}

In this paper we present the Multitemporal Adaptive Processing (MAP3) framework for the definition of a new family of multitemporal, user-oriented products whose information level lays between those of the already available Level 1 and Level 2 products. This framework is organized in three blocks of activities dealing with pre-processing, adaptive processing and representation. Experiments performed on semiarid and temperate datasets testify the reliability of the proposed framework and its independence from the sensor and the scenario.

\section{INTRODUCTION}

The increasing availability of high resolution data with short revisit time makes very attractive to operate with multitemporal datasets for application of environmental monitoring and the study of dynamic phenomena. However, the analysis of a series of images introduces relevant challenges regarding the extraction, interpretation and representation of information.

In this paper we present the Multitemporal Adaptive Processing (MAP3) [1] framework for the definition of a new family of multitemporal, user-oriented products we called Level $1 \alpha$ because they conceptually are an evolution of Level 1 products but not properly Level 2 ones.

MAP3 defines a reliable processing which should allow the achievement of a set of comparable images and the best level of of interaction between the user and the machine during the decisional phase.

The choice of appropriate color image spaces is needed in order to obtain new products that are easy to interpret for human and automatic analysis. In the past, RGB composition has been widely used because it provides products easily interpretable by human inspection [2].

A literature review about multitemporal SAR issues showed the necessity of building general and analyst-driven frameworks restoring the centrality of the users in the processing chain [3] which is usually neglected at advantage of a (often excessively) refined mathematics. Hence, the design of a new framework should be guided by the six properties of reproducibility, automation, adaptability, reversibility, visualization and interpretation. This ensures the suitability of the proposed framework with operative scenarios.

The paper is organized as follows. In Section 1 MAP3 framework is briefly discussed. Multitemporal Level $1 \alpha$ are presented in Section 4 with experimental results concerning datasets acquired on semi-arid Burkina Faso and temperate regions. Conclusions are drawn at the end of the work.

\section{MAP3 PROCESSING CHAIN}

The technique we propose for the elaboration of the new multitemporal products can be organized in three blocks of activities, as shown in the block diagram of Figure 1. The first block is related with the pre-processing chain in which data coregistration, despeckling and calibration issues are implemented. The second block concerns the adaptive processing in which the image combination which better emphasizes the relevant characteristics of the scene is chosen. Finally, the last block is relevant to representation and analysis. The design of the blocks pointed to the simplicity, minimizing the operations necessary to obtain the products, and to the availability of the algorithms. Innovation was provided in the crosscalibration step in which we introduced the Variable Amplitude Levels Equalization (VALE) method presented in Section $3.3[1]$.

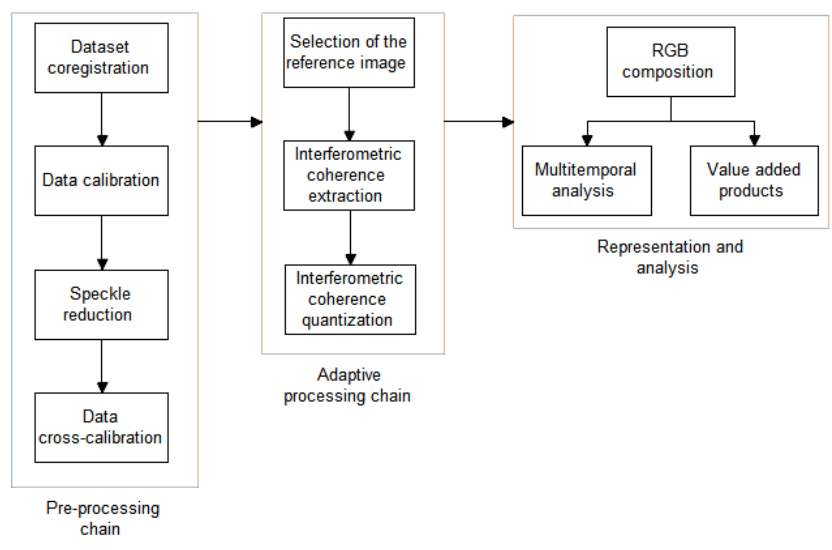

Fig. 1: MAP3 framework block diagram

In the following sections we briefly discuss the processing chain depicted in Figure 1. 


\subsection{Calibration}

One of the major challenges of the processing chain is to create a series of radiometrically comparable images. In this work we processed time series of COSMO-SkyMed and TerraSAR-X Single Look Complex images, for which the sigma naught can be evaluated applying a calibration factor calculated from ancillary data.

If the calibration of the time series is judged to be not satisfactory for the specific application, a calibration refinement can be carried out using an image-based calibration method such as PSCal [4] which is based on the retrieval of a suitable set of Permanent Scatterers to be exploited for the calculation of a calibration factor with respect to a reference image.

\subsection{Despeckling}

In multitemporal analysis, despeckling assumes a key importance, since the detrimental effects of speckle presence should significantly alter the discrimination of the features along the temporal axis. In this work we employed the multitemporal De Grandi filter which allowed a significant speckle reduction with no loss in spatial resolution (see Figure 2).

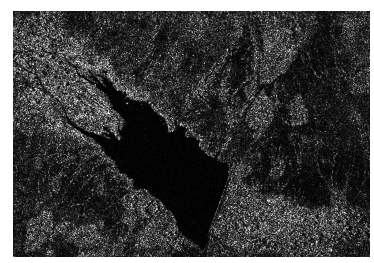

(a)

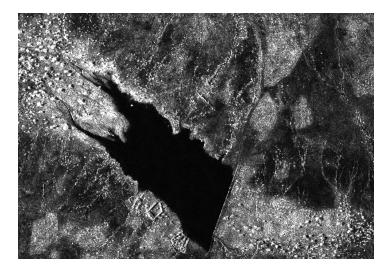

(b)
Fig. 2: Laaba basin, Burkina Faso, intensity map before (a) and after (b) the application of the De Grandi filter on a stack of 15 images.

\subsection{Cross-calibration}

In order to combine the images of the time series in a color composite view it is necessary that data are expressed in a common scale with a number of levels (usually 256) suitable with human visual perception. In the past literature (see [5]) this problem was solved with a percentile-based histogram clipping and a rescaling on 256 levels. If the scene is subject to extreme variation (as happens in semi-arid regions) this method could lead to an alteration of the amplitude ratios between the images of the time series and then to a misclassification of the relevant features of the scene.

The VALE method ensures that the histogram clipping is performed at the same amplitude level for all the elements of the time series. Under the hypothesis that the calibration step described in Section 3.1 produces perfectly calibrated images, the VALE method guarantees the balance of the channels involved in the RGB composition.
To prove this claim consider the scatter plot shown in Figure 3 in which the statistics of ten samples homogeneous areas occupied by trees taken in two different acquisitions are shown. Trees are almost stable object, thus their response is expected to be stable. This happens if the VALE method is applied. But it does not if the percentile clipping previously proposed in literature is used. This leads to an unbalance of the final RGB composition.

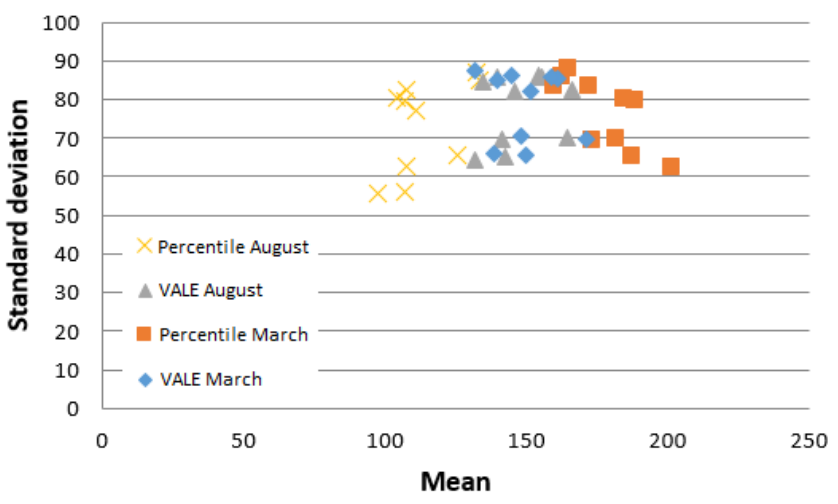

Fig. 3: Scatter plot mean VS std of ten homogeneous trees samples taken in two different acquisitions

\subsection{Adaptive processing}

The key aspect for evidencing a desired feature is the identification of a reference scenario. In semi-arid regions, it occurs at the end of the dry season (usually in April), when terrains are almost completely bare and the ponds are empty [6]. Therefore, it is possible to detect the features of interest by comparing an image acquired in a date under test (test image) with the reference image.

In the case of temperate regions the selection of the reference situation in not straightforward and is dependent on the the application the analyst is facing. For example, if summery crops has to be monitored, a reasonable choice for the reference image should be the adoption of a wintery acquisitions.

The usage of the interferometric coherence as third band allows to discriminate the presence of small human settlements.

\section{MULTITEMPORAL RGB PRODUCTS}

\subsection{Application in semi-arid environment}

The multitemporal products here presented have been obtained processing a multitemporal series of 15 COSMOSkyMed stripmap images with 3 meters of spatial resolution acquired on semi-arid Burkina Faso.

All the multitemporal products presented in the following have been obtained loading on the red, green and blue band the coherence map, the test image and the reference image, 


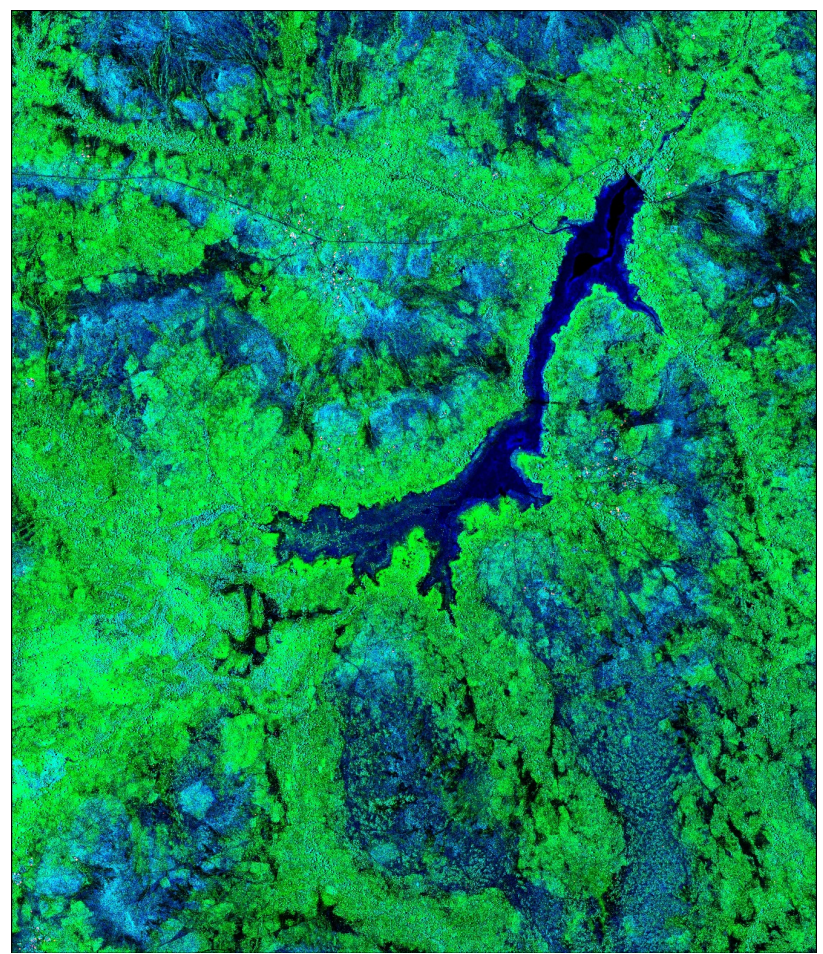

(a)

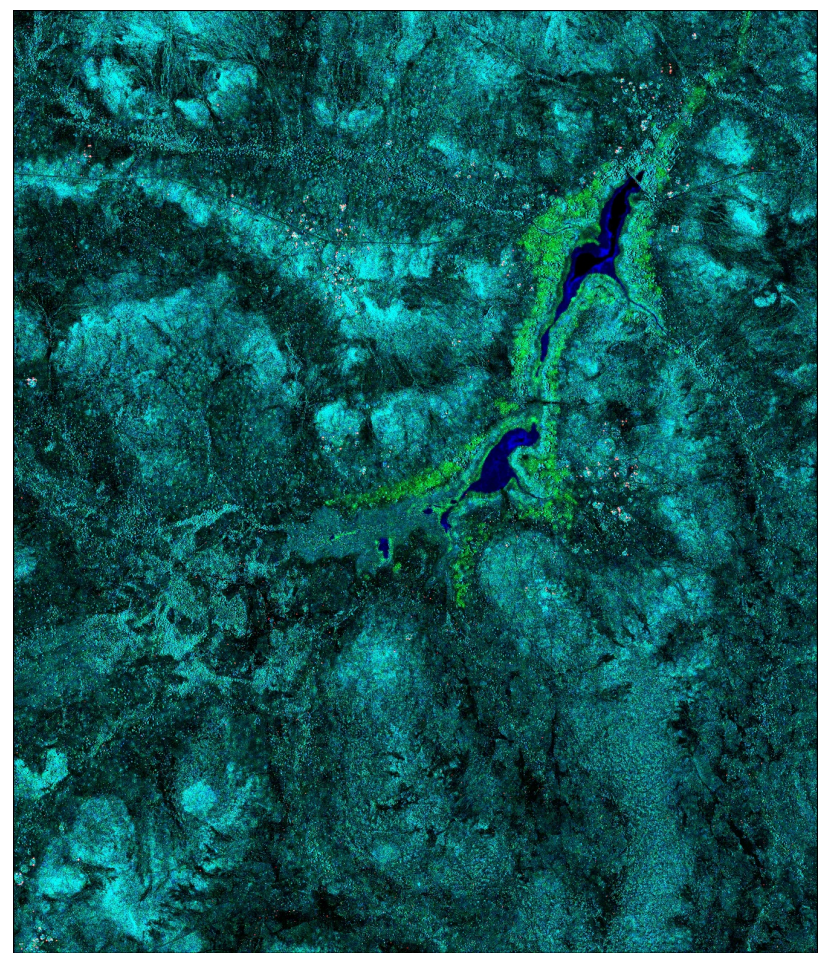

(b)

Fig. 4: COSMO-SkyMed Level $1 \alpha$ products of the study area. Red band: coherence map, Blue band: April 2011 intensity map, Green band: August 2010 intensity map (a) and March 2011 intensity map (b). The covered area is approximately $15 \times 17 \mathrm{~km}$.

respectively. The band-color association has been chosen in order to facilitate the association between the displayed colors and the physical characteristics of the scene. In Figure 4a and Figure $4 \mathrm{~b}$ the blue band is associated with the presence of water and the green band is related to the presence of vegetation. In Figure 4a the test image was acquired in August and loaded on the green band. The growth of vegetation provokes an enhancement of the backscattering in August. Hence, the green band amplitude is dominant with respect to the red and blue bands. Areas covered by seasonal surface water appear in blue because in the wet season the basins are filled up by intense rainfalls, the water acts as a reflector, and the corresponding backscattering (green band) is significantly weaker than to that of the reference dry situation (blue band). Persistent water appears as a black area within the basin, because of the low electromagnetic response both in the dry and in the wet season.

The product depicted in Figure $4 \mathrm{~b}$ is obtained through a composition of two images belonging to the dry season. The test image (green band) is acquired on March. In this case, the cyan is the dominant color because the electromagnetic response of the two scenes is almost unchanged, except for the areas in which the counter-season agriculture is exercised. Therefore, those areas are characterized by green pixels. Similarly, blue color is related with seasonal water.

In Figure 5 the reader should appreciate the level of detail achieved by the Level $1 \alpha$ product. It is also remarkable the presence of small human settlements in the neighborhood of the water source depicted as bright targets. In general, the enhancement in the interpretability of the proposed product is evident.

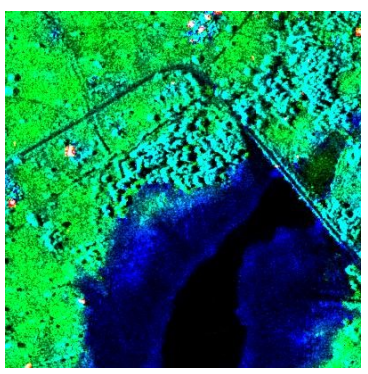

(a)

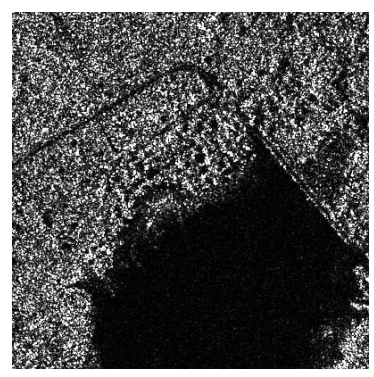

(b)
Fig. 5: Detail of the Level $1 \alpha$ (a) and SLC (b) products.

\subsection{Application in temperate areas}

In this section we want to stress the independence of MAP3 from both sensor and scenario.

The solution presented in Figure 6 has been built processing a set of TerraSAR-X stripmap acquired on Calabria, Italy. The reference image (blue band) is an acquisition of January 


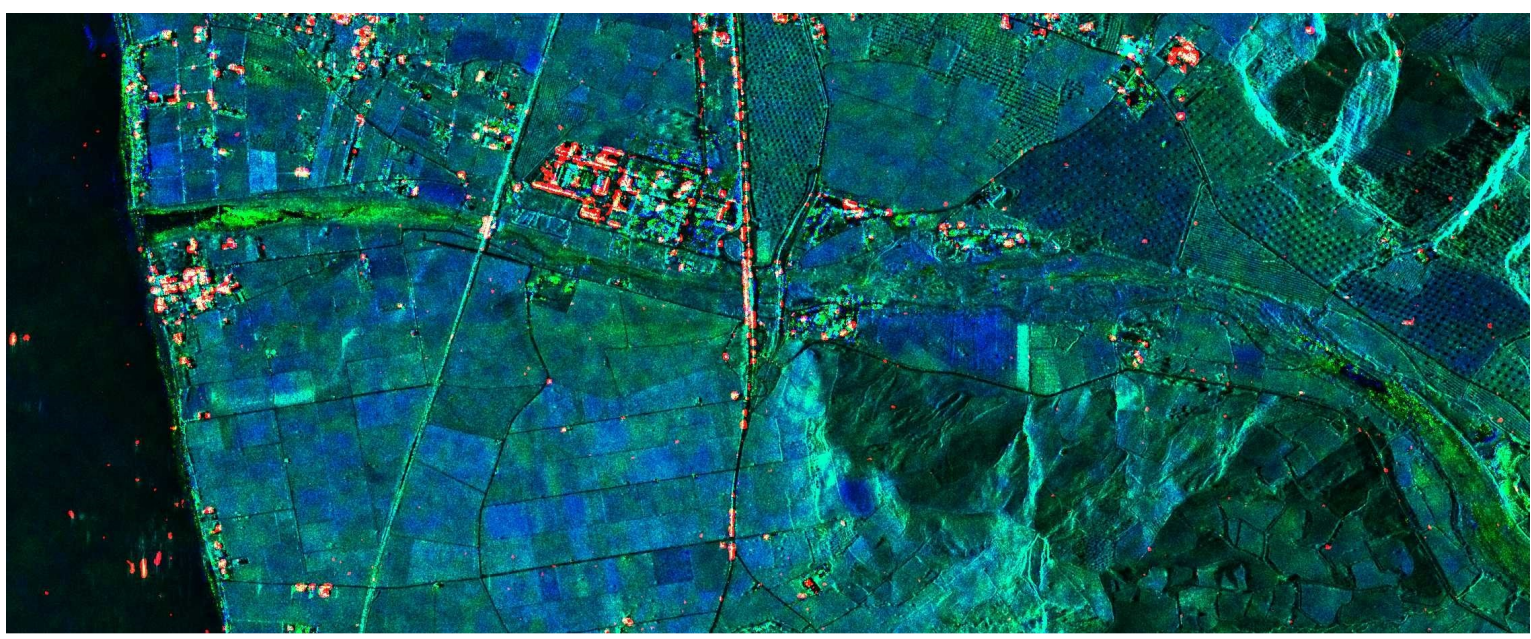

Fig. 6: Ciró Marina, Italy, TerraSAR-X Level $1 \alpha$ product extracted from a series of 35 images. Covered area is about $21 \mathrm{~km}^{2}$.

2010, while the test image (green band) has been acquired on April 2008. In this representation, the behavior of the relevant feature of the scene is the same of that previously analyzed for the Burkina Faso scene. In particular: permanent water surfaces (e.g. the sea) appear as dark areas because of the low response of all the involved channels; urban areas are represented as bright areas due to the high contributions of both intensity and interferometric coherence; cultivated areas have a color that depends on the type of cultivation. However, because of the high degree of anthropization,in this case the agronomic/botanic interpretation is not trivial and needs to be supported by specific expertise.

\section{CONCLUSIONS}

Multitemporal SAR analysis is a powerful and attractive technique for environmental monitoring and planning. It introduces challenging issues relative to data calibration and their representation. This problem has been effectively solved by MAP3, whose design pointed to the minimization of the complexity and to the exploitation of the available algorithms. An innovation in the cross-calibration phase was introduced with the VALE method.

MAP3 is robust and flexible. In fact, it can be applied in different climatic zones and for different sensors. The techniques embodied in each block can be substituted by others present in literature or implemented ex novo.

\section{ACKNOWLEDGMENTS}

The authors thank the Italian Space Agency (ASI) for providing the COSMO-SkyMed images under the aegis of the 2007 COSMO-SkyMed AO Project "Use of High Resolution SAR Data for Water Resource Management in Semi Arid Regions" and the German Space Agency (DLR) for providing the TerraSAR-X stack under the aegis of the project "Use of SAR-satellite data to monitor and model landslides and subsidence hazards".

\section{REFERENCES}

[1] D. Amitrano, G. Di Martino, A. Iodice, D. Riccio, and G. Ruello, "A New Framework for Multitemporal SAR RGB Representation: Rationale and Products," IEEE Trans. Geosci. Remote Sens., In press.

[2] D. Amitrano, G. Di Martino, A. Iodice, D. Riccio, G. Ruello, F. Ciervo, M. N. Papa, and Y. Koussoube, "Synthetic Aperture Radar for Humanitarian Purposes: Products and Opportunities," in IEEE Global Humanitarian Technology Conf., 2013, pp. 546-551.

[3] M. Datcu and K. Seidel, "Human-Centered Concepts for Exploration and Understanding of Earth Observation Images," IEEE Trans. Geosci. Remote Sens., vol. 43, no. 3, pp. 52-59, 2005.

[4] D. D'Aria, A. Ferretti, A. Monti Guarnieri, and S. Tebaldini, "SAR Calibration Aided by Permanent Scatterers," IEEE Trans. Geosci. Remote Sens., vol. 48, no. 4, pp. 2076-2086, 2010.

[5] S. G. Dellepiane and E. Angiati, "A New Method for Cross-Normalization and Multitemporal Visualization of SAR Images for the Detection of Flooded Areas," IEEE Trans. Geosci. Remote Sens., vol. 50, no. 7, pp. 27652779, 2012.

[6] D. Amitrano, G. Di Martino, A. Iodice, D. Riccio, G. Ruello, M. N. Papa, F. Ciervo, and Y. Koussoube, "Effectiveness of high-resolution SAR for water resource management in low-income semi-arid countries," Int. J. Remote Sens., vol. 35, no. 1, pp. 70-88, 2014. 